\title{
DÉPASSER LA LIMITE DE DIFFRACTION EN MICROSCOPIE DE FLUORESCENCE
}

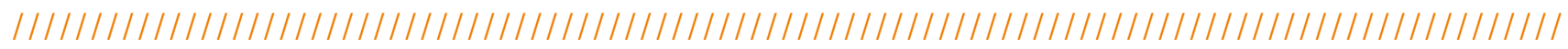

\section{Pierre Jouchet $^{1}$, Abigail llland ${ }^{1}$, Guillaume Dupuis ${ }^{1}$, Emmanuel Fort ${ }^{2}$, Sandrine Lévêque-Fort ${ }^{1}$}

${ }^{1}$ Institut des sciences Moléculaires d'Orsay, Université Paris Saclay, CNRS, 91405 Orsay

${ }^{2}$ Institut Langevin, ESPCI, Université PSL, 75005 Paris

*sandrine.leveque-fort@universite-paris-saclay.fr
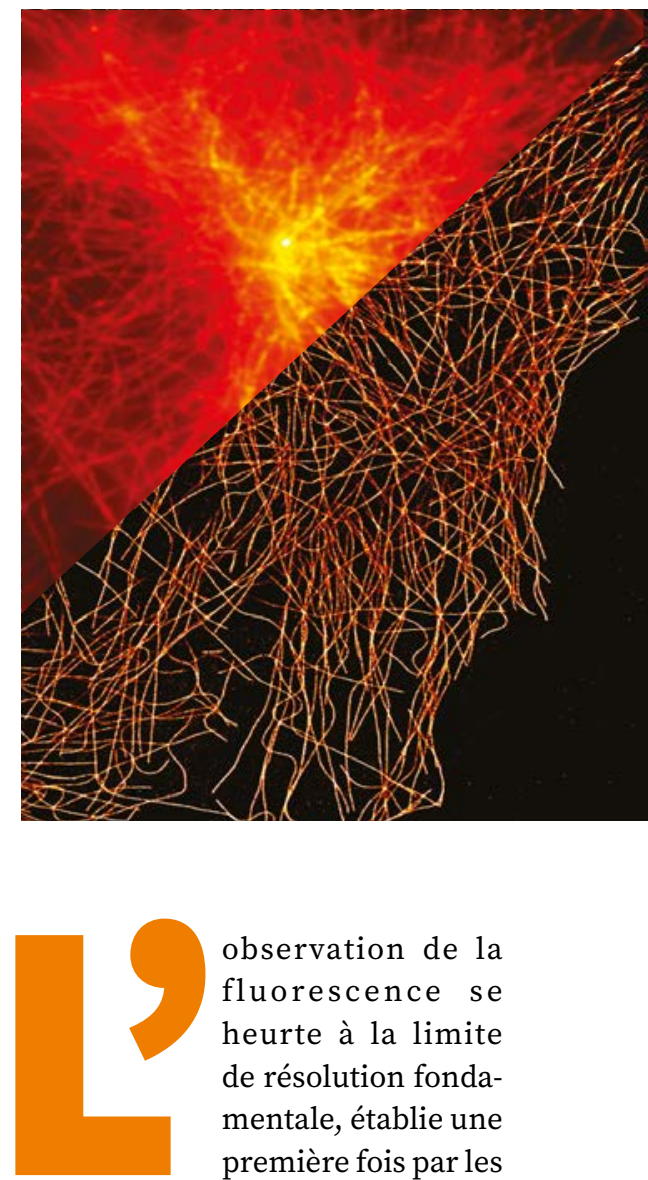

observation de la

fluorescence se heurte à la limite de résolution fondamentale, établie une première fois par les travaux de l'opticien allemand Ernst Abbe, et est imposée par la réponse du microscope à un émetteur ponctuel, qu'on appelle aussi fonction d'étalement du point (ou PSF pour Point Spread Function). L'objectif étant de pupille circulaire, la taille transverse de la PSF est donnée par $\mathrm{r}=1.22 \lambda / 2 \mathrm{ON}$, et sa taille axiale par $\mathrm{z}=2 \mathrm{n} \lambda / \mathrm{ON}^{2}$ (avec ON l'Ouverture Numérique de l'objectif et $\mathrm{n}$ l'indice de réfraction de l'échantillon). Ainsi pour un objectif d'ouverture numérique de 1,49 et une longueur d'onde

La microscopie de fluorescence est un outil de référence dans l'étude des systèmes biologiques, alliant la spécificité offerte par la fluorescence et la possibilité d'un suivi non invasif en milieu vivant. Cependant comme l'ensemble des techniques de microscopie, elle est soumise au phénomène de diffraction introduit par l'objectif, qui limite la résolution de l'instrument. Ce texte présente les différentes méthodes permettant de dépasser cette contrainte en associant développement instrumental en optique et contrôle de la photophysique des fluorophores afin de révéler l'organisation cellulaire à l'échelle nanométrique.

\section{https://doi.org/10.1051/photon/202110845}

Article publié en accès libre sous les conditions définies par la licence Creative Commons Attribution License CC-BY (https://creativecommons.org/licenses/by/4.0), qui autorise sans restrictions l'utilisation, la diffusion, et la reproduction sur quelque support que ce soit, sous réserve de citation correcte de la publication originale.

de $650 \mathrm{~nm}$, la PSF se présentera sous la forme d'un volume ellipsoïdal de taille $\sim 266 \mathrm{~nm} \times \sim 778 \mathrm{~nm}$, à l'intérieur duquel il sera impossible de distinguer différentes entités cellulaires (cf Fig.1).

On distingue deux modalités d'acquisition en microscopie de fluorescence : en balayage point par point ou en plein champ, pour lesquelles différentes stratégies ont été mises en place afin d'améliorer légèrement la résolution des images. La microscopie confocale à balayage laser repose sur l'utilisation d'un faisceau laser focalisé pour l'excitation et le positionnement d'un trou de filtrage devant le détecteur offrant un gain en résolution spatiale d'un facteur $\sqrt{ } 2$. En microscopie plein champ, l'utilisation des ondes évanescentes à l'excitation (TIRF pour Total Internal Reflection Fluorescence) ou à la détection (SAF, pour Supercritical Angle Fluorescence) permet d'introduire un sectionnement axial de l'ordre de $150 \mathrm{~nm}$. La technique SIM (Structured Illumination Microscopy) [1] utilise une illumination structurée afin de bénéficier à la fois d'un sectionnement optique en profondeur et d'une amélioration de la résolution latérale (cf Fig.2). En effet, en microscopie classique, la fonction de transfert de l'objectif filtre les fréquences spatiales détectables et notamment les hautes fréquences associées aux détails fins de l'échantillon. Le principe de la microscopie SIM est basé sur l'apparition des franges de Moiré 
produites par les interférences entre le motif structuré d'illumination et les fréquences spatiales contenues dans l'échantillon. En particulier, les franges de Moiré encodent le battement fréquentiel entre la haute fréquence spatiale de l'illumination et les très hautes fréquences spatiales de léchantillon. Ainsi la transposition par battement des fréquences hautes à une fréquence spatiale plus basse, non coupée par l'objectif, permet de bénéficier d'un domaine fréquentiel plus grand pour reconstruire l'image. Une modulation temporelle de l'excitation induite par un déplacement des franges sur l'échantillon ainsi qu'une rotation de l'excitation permet ainsi d'obtenir suffisamment d'informations pour extraire les fréquences hautes de l'échantillon dans les différentes directions. Cependant la connaissance spatiale exacte du motif d'excitation ainsi que son déplacement précis sont deux conditions nécessaires pour repositionner les fréquences hautes de l'échantillon dans la nouvelle fonction de transfert qui nécessite une phase de traitement numérique particulièrement délicate. De plus, la fréquence du motif d'illumination et celle de la figure de Moiré restant inférieures à la fréquence de coupure du microscope, les techniques SIM ne permettent une amélioration de la résolution spatiale que d'un facteur 2. Elles ont en revanche l'avantage d'être parfaitement compatibles avec l'observation d'échantillons vivants.

Ainsi, si différentes stratégies optiques ont conduit à une amélioration limitée de la résolution, il a fallu attendre l'alliance de nouvelles approches optiques et d'une ingénierie des transitions moléculaires pour que la limite de diffraction soit complétement dépassée, offrant ainsi accès au milieu biologique à l'échelle nanoscopique. Le prix Nobel de Chimie 2014 a récompensé ces deux nouvelles familles de techniques d'imagerie qui ont permis de dépasser la limite de diffraction via (i) l'observation

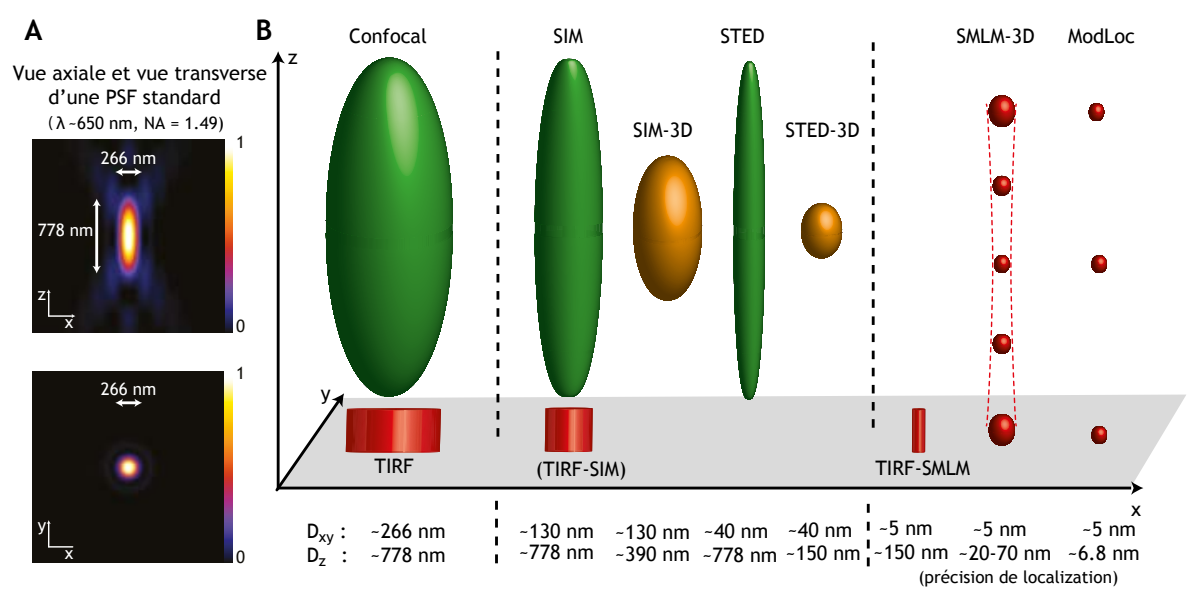

Figure 1.

A) Vue axiale et transversale de la PSF. B) Comparatif des tailles de PSF pouvant être obtenues par les différentes méthodes d'imagerie au-delà de la limite de diffraction.

de molécules uniques - on parle alors de Single Molecule Localization Microsopy (SMLM) (W.E. Moerner et E. Betzig [2-3]) - et (ii) en confinant l'émission de fluorescence grâce à la microscopie STED (Stimulated Emission Depletion) (S. Hell [4]). Ces stratégies reposent sur le contrôle - respectivement stochastique et déterministe - du nombre de fluorophores émettant simultanément de la lumière dans le volume focal à un instant donné. Les techniques déterministes comme la microscopie STED exercent un contrôle spatial afin de forcer les molécules à la périphérie du volume focal à revenir à l'état fondamental par émission stimulée et ainsi limiter au centre du volume focal les molécules pouvant émettre de la fluorescence. L'image super-résolue est donc obtenue en balayant ce volume d'émission spontanée restreint. Les techniques stochastiques de localisation de molécules uniques (SMLM), réalisent ce contrôle de façon temporelle : à un instant donné, seul un fluorophore positionné aléatoirement dans le volume focal émet de la fluorescence. L'ensemble des photons d'émission spontanée mesurés dans ce volume sont issus d'une seule molécule individuelle qui peut être ainsi localisée par analyse de son image avec une précision nanométrique. L'image super-résolue est obtenue en mesurant séquentiellement la position des différentes molécules fluorescentes.

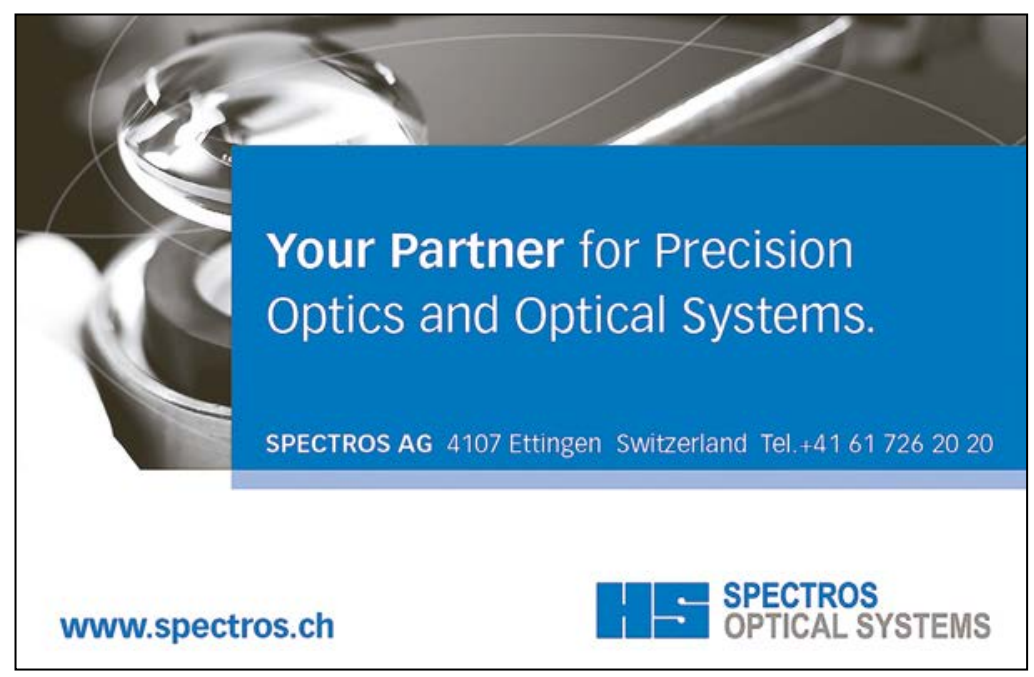



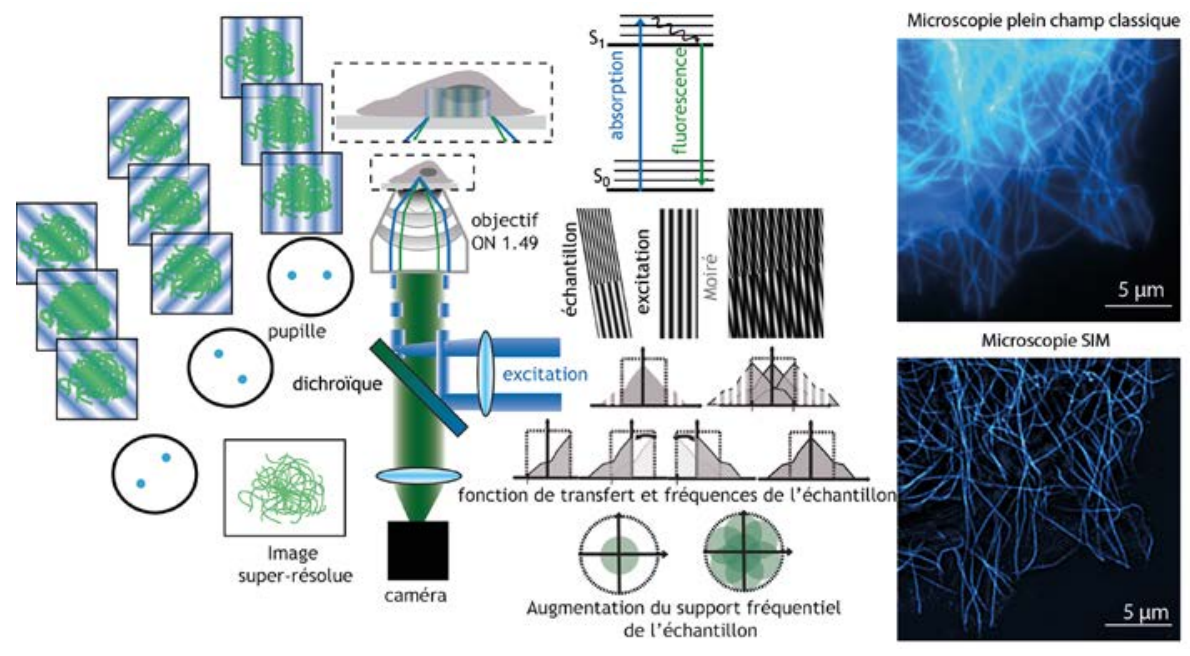

Microscopie SIM

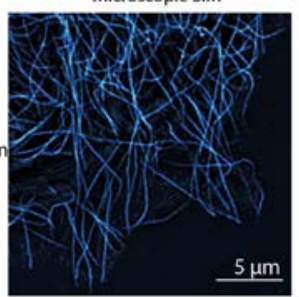

Le concept de la microscopie STED a été proposé dès 1994 [4] par l'équipe de $\mathrm{S}$. Hell mais n'a concrètement été démontré expérimentalement qu'à partir de 1999 du fait de sa complexité expérimentale (cf Fig.3). En effet, superposé au faisceau d'excitation balayé sur l'échantillon, un deuxième faisceau permet d'induire de façon spatialement sélective la déplétion du niveau excité par émission stimulée et ainsi de réduire la zone pouvant émettre de la fluorescence. La mise en forme de ce faisceau STED est extrêmement délicate et requiert un excellent contrôle des différents éléments optiques et de la polarisation du faisceau afin d'obtenir une intensité centrale nulle au centre permettant l'émission de fluorescence. De façon courante, le faisceau STED présente une forme toroïde permettant de confiner l'émission spontanée uniquement dans le plan transverse à l'axe optique. Un confinement suivant l'axe optique peut également être obtenu par superposition de faisceaux STED suivant différentes orientations afin de bénéficier d'un confinement en 3 dimensions. La longueur d'onde du faisceau STED étant choisie en général en bord du spectre d'émission du fluorophore observé, l'émission stimulée peut être ainsi facilement spectralement discriminée de l'émission de fluorescence. Les faisceaux d'excitation et de déplétion restant limités par la

\section{Figure 2.}

Principe de la microscopie SIM qui est compatible avec l'ensemble des fluorophores : l'échantillon est éclairé en champ large et pour différentes positions et orientations du motif d'excitation, ce qui permet de transposer les fréquences hautes de l'échantillon et de les intégrer à l'image finale super-résolue. Exemple d'images sur des tubulines de cellules COS7.

\section{Figure 3.}

Principe de la microscopie STED : L'échantillon est sondé point par point par la superposition d'un faisceau d'excitation et du faisceau de déplétion mis en forme et réduisant le volume autorisé à émettre de la fluorescence. Exemple d'images sur des tubulines (bleu) et vésicules de clathrine (jaune) de cellules COS7 (échelle $5 \mu \mathrm{m}$ ).

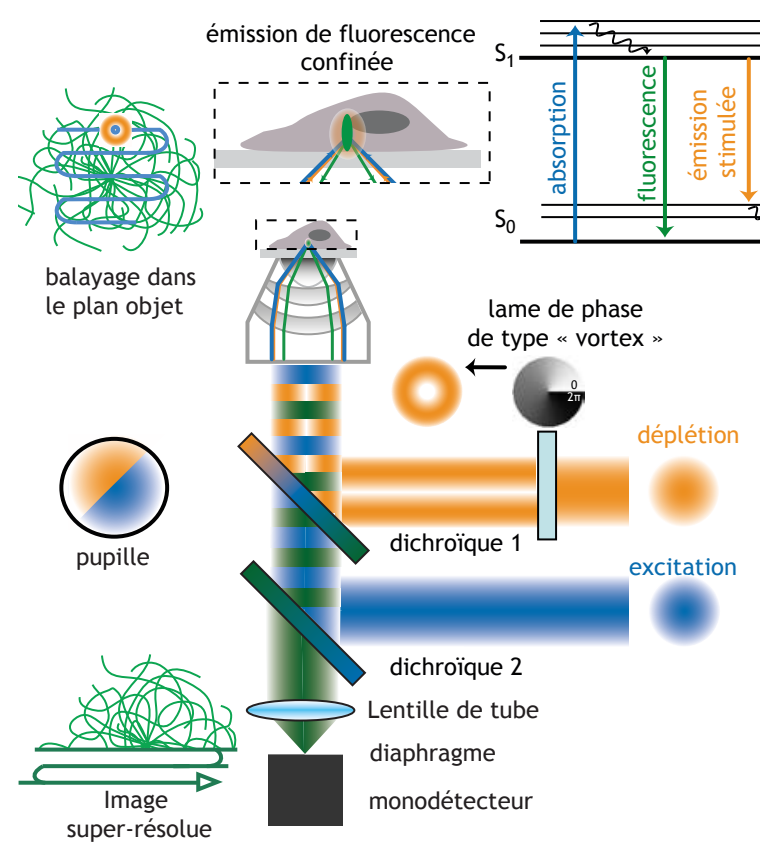

diffraction, l'augmentation de l'intensité du faisceau STED permet de saturer la déplétion de l'état excité afin de réduire la partie centrale non déplétée à une zone de l'espace non limitée par la diffraction. Si en théorie le gain en résolution spatiale est seulement limité par la puissance du faisceau STED, en pratique il est conditionné par des puissances permettant d'atteindre 30-40 $\mathrm{nm}$ de résolution latérale afin d'éviter les photodégradations de l'échantillon. La microscopie STED permet d'obtenir directement l'image super-résolue, sans nécessité de post-traitement, et elle est compatible avec des observations en échantillon vivant. Cependant, l'observation à grande profondeur peut nécessiter l'utilisation de boucles d'optique adaptative afin de conserver la mise en forme particulière du faisceau STED.

Les approches de localisation de molécules permettent d'atteindre des résolutions encore supérieures, et bénéficient des différentes stratégies mises en place pour le suivi de trajectoire d'objets uniques depuis plus de 20 ans. L'image d'une molécule fluorescente isolée correspond à une tache limitée par la diffraction dont le barycentre

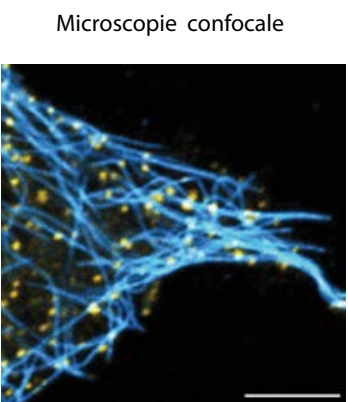

Microscopie STED

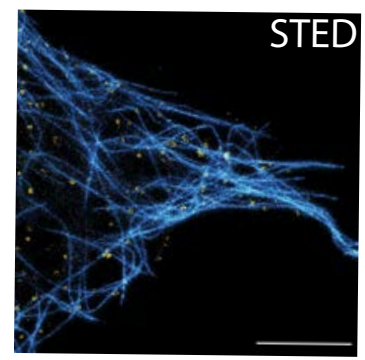


est confondu avec la position de la molécule. Cette dernière peut alors être localisée avec une précision de quelques nanomètres, typiquement inversement proportionnelle à $\sqrt{N}$, où $N$ est le nombre de photons émis par la molécule. Comment peut-on étendre ce concept à l'observation d'échantillons biologiques densément marqués en fluorophores ? L'enjeu est de trouver une astuce pour contraindre les molécules fluorescentes à ne pas émettre toutes en même temps afin de permettre la localisation de chacune d'entre elles de façon décalée dans le temps et l'espace. La première approche repose sur l'utilisation de protéines fluorescentes photo-activables (PALM pour Photo-Activated Light Microscopy) où une sous-population peut être photo-activée ou photo-convertie par application d'une très faible excitation à 405 $\mathrm{nm}$. L'approche STORM (Stochastic Optical Reconstruction Microscopy) quant à elle repose sur l'utilisation de fluorophores organiques, couramment utilisés en microscopie de fluorescence conventionnelle, immergés dans une solution tampon d'oxydo-réduction. Dans cette solution, les fluorophores cyclent continûment entre un état singulet

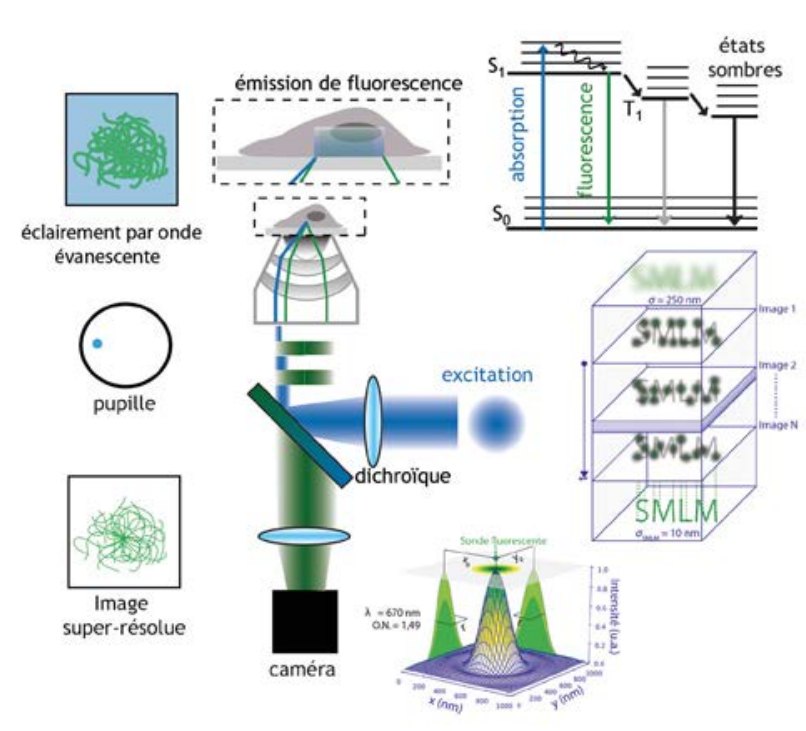

Microscopie plein champ classique

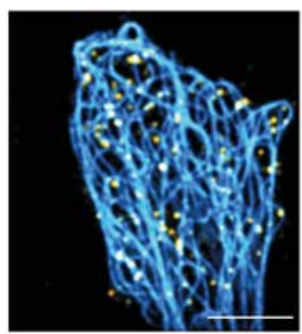

Microscopie SMLM

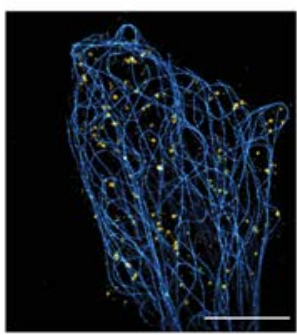

Figure 4.

Principe de la microscopie SMLM : L'échantillon est soumis à un éclairement en champ large intense afin de favoriser le passage des fluorophores vers les états sombres et permettant ainsi l'observation de molécules individuelles pouvant être localisées. Exemple d'images sur des tubulines (bleu) et vésicules de clathrine (jaune) de cellules COS7 (échelle $5 \mu \mathrm{m}$ ).

$\mathrm{S}_{1}$ fluorescent et des états sombres non-fluorescents métastables, permettant la localisation successive des molécules. L'avantage des fluorophore organiques repose sur le nombre de photons émis plus élevés ( 5000 photons par molécule) permettant une localisation plus précise qu'en PALM ( $~ 500$ photons). Une dernière famille, appelé PAINT (Point Accumulation for Imaging in Nanoscale Topography), repose sur la diffusion de sondes fluorescentes dans l'échantillon. Grâce à une affinité chimique, elles s'accrochent de manière transitoire aux sites spécifiquement marqués et peuvent ainsi être observées durant le temps d'intégration avant de se détacher du site d'intérêt. L'une des catégories de PAINT les plus utilisées

\section{small components MASSIVE IMPACT}

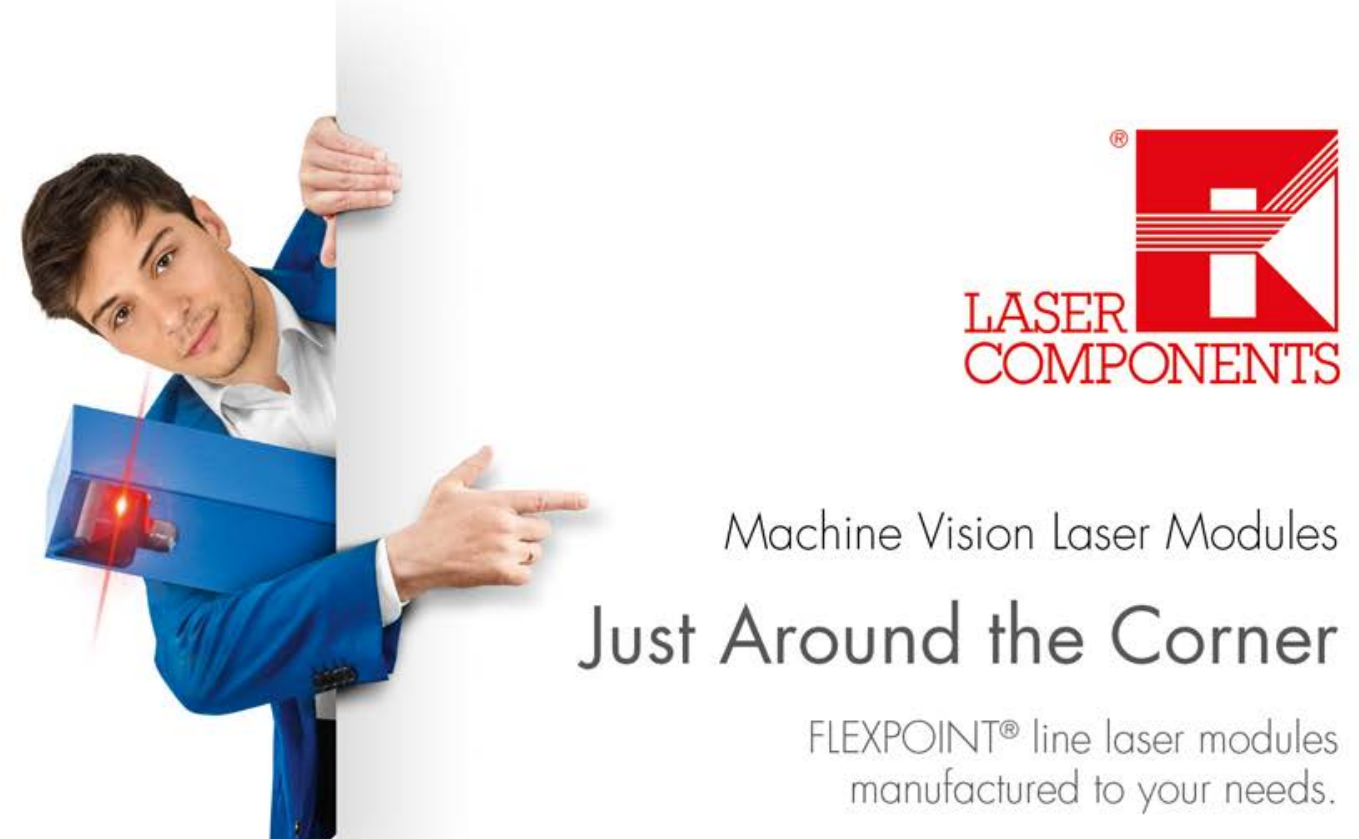

www. lasercomponents.fr 
A
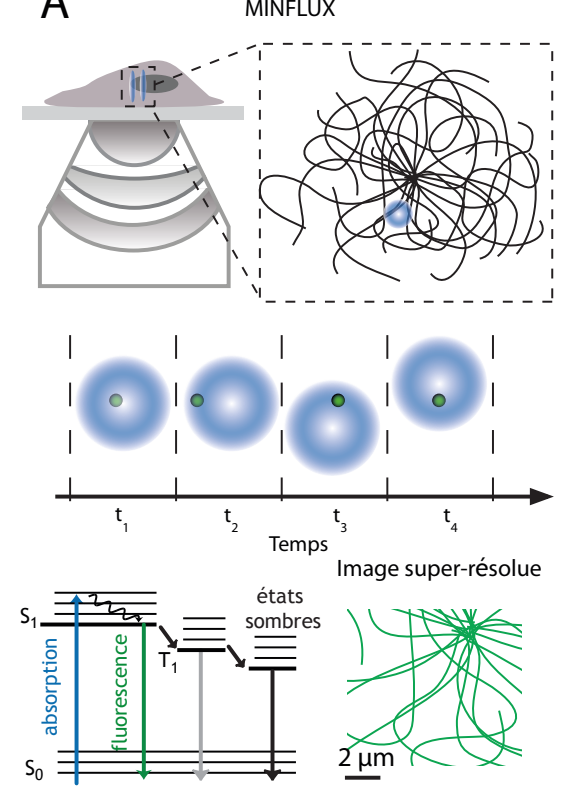

B
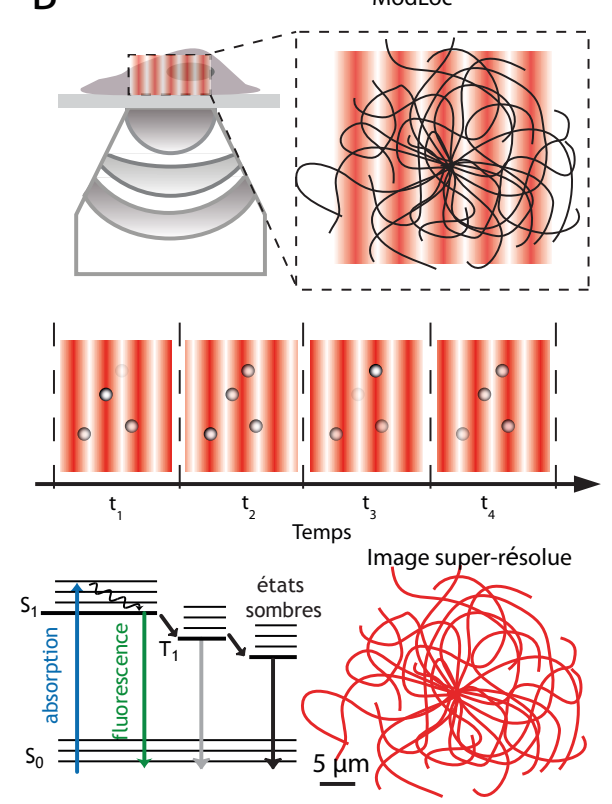

Figure 5. Nouveaux concepts permettant d'améliorer la localisation des molécules uniques A) approche point par point où chaque molécule est détectée via une séquence de position du faisceau mis en forme, et offrant un champ de vue réduit B) approche champ large, l'ensemble des molécules est soumis à la même séquence d'excitation structurée induisant une modulation temporelle de la fluorescence.

associer les déformations induites à des positions axiales qui s'estompent très vite lors d'observation en profondeur. D'autres familles de mises en forme de PSF ont été développées afin d'étendre les performances. L'utilisation de la lumière supercritique peut également apporter intrinsèquement cette information axiale [5].

\section{CONCLUSION}

Ces différentes familles de super-résolution, SIM, STED, SMLM offrent donc des contraintes expérimentales et d'analyses différentes ainsi que des performances complémentaires en termes de profondeur d'observation et de résolutions latérale et axiale, permettant de dépasser la limite de diffraction. Alors qu'elles semblaient évoluer chacune dans leur direction, des récents travaux ont proposé d'associer ces techniques afin de réaliser l'étape de localisation des molécules uniques via l'introduction d'une illumination structurée permettant de déterminer la position des molécules grâce à la modulation temporelle de la fluorescence (cf Fig.5). En champ large, cette approche a été récemment proposée pour améliorer la résolution axiale (ModLoc[6]) où une précision axiale sous les $7 \mathrm{~nm}$ peut être obtenue en profondeur, ou latérale permettant d'obtenir un gain d'un facteur 2 (SIMPLE, Simflux, Rose). Associée à une approche bénéficiant $\mathrm{du}$ savoir-faire de mise en forme $\mathrm{du}$ faisceau STED mais pour induire cette fois une excitation non-uniforme, la localisation des molécules uniques se fait via une séquence de positions du faisceau dédiée à chaque molécule (Minflux [7]), comme un nano-GPS, où legainaccru en résolution axiale $(\sim 2 \mathrm{~nm})$ se fait au détriment d'un champ de vue très restreint (quelques $\mu \mathrm{m}^{2}$ ). La convergence de ces approches ouvre ainsi de nouveaux horizons qui permettront de continuer à repousser les limites de la diffraction. solue finale en fin d'acquisition. Ce processus de localisation ne donnant accès qu'à une information spatiale transverse, des stratégies complémentaires doivent être mises en place afin de localiser la position des molécules suivant l'axe optique. La plus commune repose sur l'introduction d'une lentille cylindrique devant la caméra, qui induit une déformation de la PSF permettant d'identifier la position axiale de la molécule. Simple à mettre en œuvre, cette approche nécessite une calibration axiale rigoureuse pour

\section{RÉFÉRENCES}

[1] M. G. Gustafsson, J. Microsc. 198, 82-87 (2000)

[2] R. M. Dickson, A. B. Cubitt, R. Y. Tsien, W. E. Moerner, Nature 388, 355-358 (1997)

[3] E. Betzig, G. H. Patterson, R. Sougrat et al., Science 313, 1642-1645 (2006).

[4] S. W. Hell, J. Wichmann, Opt. Lett. 19, 780-782 (1994).

[5] N. Bourg, C. Mayet, G. Dupuis et al., Nat. Photonics 9, 587-593 (2015)

[6] P. Jouchet, C. Cabriel, N. Bourg et al., Nat. Photonics 15, 297-304 (2021)

[7] F. Balzarotti, Y. Eilers, C. Gwosch, A. H. Gynna et al., Science 355, 606-612 (2017) 